\title{
An open-label clinical trial to investigate the efficacy and safety of corifollitropin alfa combined with hCG in adult men with hypogonadotropic hypogonadism
}

Eberhard Nieschlag ${ }^{1 *}$ (D), Pierre-Marc G. Bouloux², Barbara J. Stegmann ${ }^{3}$, R. Ravi Shankar³, Yanfen Guan³, Anjela Tzontcheva ${ }^{3}$, Christine McCrary Sisk ${ }^{3}$ and Hermann M. Behre ${ }^{4}$

\begin{abstract}
Background: Hypogonadotropic hypogonadism $(\mathrm{HH})$ in men results in insufficient testicular function and deficiencies in testosterone and spermatogenesis. Combinations of human chorionic gonadotropin ( $\mathrm{hCG}$ ) and recombinant follicle-stimulating hormone (recFSH) have been successful in the treatment of $\mathrm{HH}$. Corifollitropin alfa is a long-acting FSH-analog with demonstrated action in women seeking infertility care. The aim of this study was to investigate the efficacy and safety of corifollitropin alfa combined with hCG to increase testicular volume and induce spermatogenesis in men with $\mathrm{HH}$.
\end{abstract}

Methods: This was a Phase III, multi-center, open-label, single-arm trial of corifollitropin alfa in azoospermic men aged 18 to 50 years with $\mathrm{HH}$. After 16 weeks of pretreatment of 23 subjects with hCG alone, 18 subjects with normalized testosterone (T) levels who remained azoospermic entered the 52-week combined treatment phase with hCG twice-weekly and $150 \mu \mathrm{g}$ corifollitropin alfa every other week. The increase in testicular volume (primary efficacy endpoint) and induction of spermatogenesis resulting in a sperm count $\geq 1 \times 10^{6} / \mathrm{mL}$ (key secondary efficacy endpoint) during 52 weeks of combined treatment were assessed. Safety was evaluated by the presence of anti-corifollitropin alfa antibodies and the occurrence of adverse events (AES).

Results: Mean $( \pm \mathrm{SD})$ testicular volume increased from $8.6( \pm 6.09) \mathrm{mL}$ to $17.8( \pm 8.93) \mathrm{mL}$ (geometric mean fold increase, 2.30 [95\% Cl: 2.03, 2.62]); $14(77.8 \%)$ subjects reached a sperm count $\geq 1 \times 10^{6} / \mathrm{mL}$. No subject developed confirmed anti-corifollitropin alfa antibodies during the trial. Treatment was generally well tolerated.

Conclusions: Corifollitropin alfa $150 \mu \mathrm{g}$ administrated every other week combined with twice-weekly hCG for 52 weeks increased testicular volume significantly, and induced spermatogenesis in $>75 \%$ of men with $\mathrm{HH}$ who had remained azoospermic after hCG treatment alone.

Trial registration: ClinicalTrials.gov: NCT01709331.

Keywords: Corifollitropin alfa, Gonadotropin deficiency, Hypogonadotropic hypogonadism, Male infertility, Testis

\footnotetext{
* Correspondence: eberhard.nieschlag@ukmuenster.de

1 University Hospital of Muenster, Center of Reproductive Medicine and

Andrology, Domagkstraße 11, D-48149 Muenster, Germany

Full list of author information is available at the end of the article
} 


\section{Background}

Male hypogonadotropic hypogonadism $(\mathrm{HH})$ is characterized by impairment in the secretion of the pituitary gonadotropins, follicle-stimulating hormone (FSH), and luteinizing hormone $(\mathrm{LH})$, resulting in insufficient testicular function and deficiencies in testosterone $(\mathrm{T})$ and spermatogenesis $[1,2]$. The onset of $\mathrm{HH}$ prior to adolescence results in delayed or absent puberty with the respective clinical consequences, whereas onset in adult life leads to hypogonadism with loss of androgenicity and infertility $[1,2]$. The approach to treatment of $\mathrm{HH}$ in males varies based on the desired outcome; $\mathrm{T}$ or human chorionic gonadotropin (hCG) therapy alone are sufficient to induce virilization. However, treatment with gonadotropins ( $\mathrm{LH}$ and $\mathrm{FSH}$ ) or gonadotropin-releasing hormone $(\mathrm{GnRH})$ is required to improve testicular function and induce spermatogenesis $[1,2]$. The hormone hCG has been successfully used to replace LH-activity since the 1950s. For FSH-activity, human menopausal gonadotropin (hMG) became available in the 1960s [3, 4] before recombinant FSH was synthesized in the 1990s and became the standard therapy for replacement in $\mathrm{HH}$ patients for stimulation of spermatogenesis [5]. In a previous clinical trial of recombinant FSH (recFSH; Puregon ${ }^{\circ}$ ) in men with $\mathrm{HH}(N=30)$, hCG in combination with recFSH resulted in a doubling of the combined testicular volume from 11.4 to $24.0 \mathrm{~mL}$, and achievement of sperm counts $\geq 1 \times 10^{6}$ in 14 of 30 (47\%) men who had remained azoospermic after $\mathrm{T}$ levels had been normalized during 16 weeks of pretreatment with hCG alone [6]. However, due to the short half-life of FSH, recFSH needs to be injected 3 times per week for extended periods of several months to years.

Corifollitropin alfa (MK-8962; formerly Org 36286/ $\mathrm{SCH}$ 900962) is a recombinant gonadotropin consisting of the $\alpha$-subunit of human FSH and a hybrid subunit composed of the sequence of the $\beta$-subunit of human FSH and the carboxy-terminal peptide part of the $\beta$-subunit of hCG. In women seeking infertility care, treatment with corifollitropin alfa produced a similar therapeutic response compared with that of recFSH [7-9]. Corifollitropin alfa has the same pharmacodynamic (PD) profile as recFSH, but an approximately two-fold longer elimination half-life $\left(t_{1 / 2}\right)$ and an almost four-fold extended time interval to peak serum levels relative to that of recFSH [10]. As a result, a single injection of corifollitropin alfa replaces 7 days of daily recFSH. Since treatment of $\mathrm{HH}$ in males usually requires long-term treatment with frequent injections of recFSH, the use of corifollitropin alfa, requiring fewer injections, may result in fewer medication errors and improved compliance.

The majority of clinical trials comparing corifollitropin alfa with daily recFSH have been conducted in women undergoing controlled ovarian stimulation for the development of multiple follicles and pregnancy for assisted reproductive technology (ART). A Phase 1, open-label trial with corifollitropin alfa conducted in 13 men with $\mathrm{HH}$ demonstrated that a single dose of $15 \mu \mathrm{g}$ corifollitropin alfa was able to induce an increase in serum inhibin-B concentrations [11]. The objective of the present study was to investigate the efficacy and safety of corifollitropin alfa in combination with hCG to increase testicular growth and induce spermatogenesis resulting in a sperm count $\geq 1 \times 10^{6} / \mathrm{mL}$ in adult men with $\mathrm{HH}$ who remained azoospermic after treatment with hCG alone.

\section{Methods}

\section{Study design and population}

This was a phase III, multi-center, open label, single-arm trial conducted in 13 centers in Australia, Germany, Italy, Poland, Spain, and the United Kingdom from February 14, 2013 to April 8, 2015 (Protocol: MK-8962031-01, formerly known as SCH 900962, P07937; ClinicalTrials.gov: NCT01709331). The study protocol was approved by the institutional review boards at every study center and informed consent was obtained before the initiation of any study procedures. The study was conducted in accordance with the principles of Good Clinical Practice and applicable country and/or local statutes and regulations regarding ethical committee review, informed consent, and the protection of human subjects participating in biomedical research.

The study design is illustrated in Additional file 1: Figure S1. The trial began at Week -16 with an hCGonly pretreatment phase, during which eligible men with $\mathrm{HH}$ were treated with 1500 IU of hCG (Merck \& Co., Inc., Kenilworth, NJ, USA) administered subcutaneously (SC) twice-weekly for 16 weeks to normalize $\mathrm{T}$ levels and to determine if hCG alone would be sufficient to achieve spermatogenesis. The dose could be increased to 3000 IU twice-weekly after 8 weeks if the Total T level remained below the unequivocal lower limits of the normal range $(8.68 \mathrm{nmol} / \mathrm{L}[250 \mathrm{ng} / \mathrm{dL}])$. Continuation into the 52-week combined treatment phase (corifollitropin alfa with hCG) was limited to subjects with normalized $\mathrm{T}$ levels who remained azoospermic at the conclusion of the pretreatment phase.

Day 1 of the trial was the first day of the 52-week combined treatment phase (corifollitropin alfa with hCG). During this phase, subjects received corifollitropin alfa $(150 \mu \mathrm{g}$ SC) once every two weeks in addition to twice-weekly hCG. The initial hCG dose in the combined treatment phase was the same as the final dose in the pretreatment phase; the dose was then adjusted up to $3000 \mathrm{IU}$ or down to $1500 \mathrm{IU}$ as needed to maintain Total $\mathrm{T}$ and estradiol (E2) levels within acceptable ranges according to the investigator's discretion. hCG was 
administered on the same two days every week (Monday/ Thursday or Tuesday/Friday), and corifollitropin alfa was given on one of the days when hCG was administered. The final follow-up visit occurred at least 21 days after the last dose of corifollitropin alfa and at least seven days after the last dose of hCG. At this visit, all subjects were assessed for any adverse events (AEs) that occurred after the administration of the last dose of trial medication. Blood samples were collected for the last assessment of hormones and anti-drug antibodies. Information was also collected on any treatment the subjects received, for either AEs or for the treatment of $\mathrm{HH}$, after the trial.

Men between 18 and 50 years, with congenital or acquired $\mathrm{HH}$, who had azoospermia, low circulating levels of gonadotropins (FSH and $\mathrm{LH} \leq 2 \mathrm{IU} / \mathrm{L}$ ) and $\mathrm{T}$ $(\leq 6 \mathrm{nmol} / \mathrm{L})$, presence of scrotal testes, adequate replacement of other pituitary hormones (if applicable), good general physical and mental health, and ability and willingness to comply with the protocol and provide written informed consent were included in the study. Important exclusion criteria included the presence of primary hypogonadism (e.g., Klinefelter syndrome), history of unilateral or bilateral cryptorchidism, and history or presence of testicular pathology of clinical importance and/or vasectomy. Men who were treated with FSH, hCG, or GnRH within the previous 3 months or for $>1$ month within the previous 6 months before signing informed consent were excluded. Other exclusion criteria were hypophysectomy within 6 months prior to screening, past or present oncologic treatment, diabetes mellitus, untreated hyperprolactinemia, uncontrolled non-gonadal endocrinopathies (thyroid, adrenal, pituitary disorders), history of alcohol or drug abuse, and administration of hormonal preparations, agents known to impair testicular function or affect sex hormone secretion, and known or suspected teratogens within 1 month before the start of screening. Prior use of androgen preparations was permitted if the patient underwent the wash-out period to bring the total $\mathrm{T}$ down to $\leq 6 \mathrm{nmol} / \mathrm{L}(173 \mathrm{ng} / \mathrm{dL})$.

\section{Endpoints}

The primary efficacy endpoint was the change from Day 1 to Week 52 (i.e., during the combined treatment phase) in log-transformed testicular volume (measured as the sum of ultrasound determination of volumes of left and right testes). The key secondary efficacy endpoint was the percentage of subjects with induced spermatogenesis resulting in a sperm count $\geq 1 \times 10^{6} / \mathrm{mL}$ at any time point at or before Week 52 . Other secondary efficacy endpoints included serum concentrations of the hormones T, E2, FSH, sex hormone-binding globulin (SHBG), inhibin B, anti-Müllerian hormone (AMH), and luteinizing hormone (LH) measured during the combined treatment phase. The primary safety endpoint was the development of anti-corifollitropin alfa antibodies.

\section{Efficacy assessments Determination of testicular volume and semen analysis}

Testicular ultrasound was performed to measure and record the maximum longitudinal, antero-posterior, and transverse diameters, and evaluate the sonographic pattern of each testis at weeks $-16,-8$, and -1 of the hCG pretreatment phase, the first day of administration of corifollitropin alfa, every 4 weeks between weeks 4 to 52 , and at discontinuation if the subject in the combined treatment phase withdrew before week 52 .

Semen samples (produced by masturbation after sexual abstinence for at least $48 \mathrm{~h}$ ) at screening, week -1 (last week of the hCG pretreatment phase and prior to start of the combined treatment phase), weeks 16, 28, 40 , and 52 , and discontinuation in subjects who withdrew prior to week 52 , were analyzed according to the WHO manual (2010) [12]. Investigational sites received training for the standard operating procedures of semen analysis prior to the start of study. Analyses for semen volume, sperm density, motility, and morphology were performed at local semen laboratories using standardized methodology across all study sites. Azoospermia was diagnosed if no spermatozoa were found in the sediment of a centrifuged sample.

\section{Endocrine parameters}

Blood samples were collected before the injection(s) of hCG or corifollitropin alfa for the measurement of T, E2, FSH, SHBG, inhibin B, AMH, and LH at weeks $-16,-8$, and -1 of the hCG pretreatment phase, the first day of administration of corifollitropin alfa, every 4 weeks between weeks 4 to 52 , at discontinuation if the subject in the combined treatment phase withdrew before week 52, and at the follow-up visit. Total T and E2 concentrations were determined centrally by Quest, Valencia, CA, USA, using liquid chromatography-mass spectrometry (LCMS). Serum FSH, SHBG, and LH concentrations were determined centrally by Analytical Biochemical Laboratory (ABL), Assen, The Netherlands, using kits for time-resolved fluoroimmunoassays (AutoDelfia, Wallac Oy, Finland). Inhibin B and AMH serum concentrations were analyzed centrally by $\mathrm{ABL}$, Assen, The Netherlands, using a validated enzyme immunoassay (EIA) kit (Gen II ELISA; Beckman Coulter, Chaska, MN, USA).

\section{Safety assessments}

Adverse events (AEs) and serious AEs (SAEs) were assessed throughout the study. Routine blood chemistry, hematology, urinalysis, physical and andrological examinations, and body weight, height, blood pressure, and 
heart rate measurements were performed throughout the study. Development of anti-corifollitropin alfa antibodies was a primary safety endpoint. Serum samples were collected for the assessment of anti-corifollitropin alfa antibodies at weeks -16 and -1 of the hCG pretreatment phase, weeks $4,16,28,40$, and 52 , at discontinuation if the subject in the combined treatment phase withdrew prior to week 52, and at the follow-up visit. All blood sampling for antibodies was performed prior to the scheduled injection(s) of hCG or corifollitropin alfa for that day. Other events of clinical interest included elevated aspartate aminotransferase (AST) or alanine aminotransferase (ALT) $\geq 3 \times$ the upper limit of normal (ULN) and elevated total bilirubin $\geq 2 \times$ ULN and simultaneous alkaline phosphatase $<2 \times$ ULN and hypersensitivity reactions (skin rash, urticaria, hypotension, allergic asthma, chest tightness, bronchospasm, dyspnea, or wheezing following injection with corifollitropin alfa).

\section{Statistical analyses}

It was initially planned that 40 to 50 subjects would be screened. Assuming a screen failure rate of 50 to $60 \%$, this would result in about 20 subjects that were expected to enter the hCG pretreatment phase. A minimum of 10 subjects with normalized $\mathrm{T}$ levels who remained azoospermic at the end of the pretreatment phase were expected to enter the combined treatment phase. Sample size estimates were based on the results from a previous trial with recFSH in $\mathrm{HH}$ men [6]. The geometric mean increase in testicular volume during 48 weeks of recFSH treatment was 1.77 or, equivalently, 0.57 on $\log$-scale (base e $=2.178$ ) with a standard deviation $(\mathrm{SD})$ of $0.36(n=16)$. Under the assumptions of a delta of 0.55 and a SD of 0.4 (both on the log-scale) and a one-sided significance level alpha of 0.025 , a total of 10 azoospermic men (with normalized T levels after 16 weeks of hCG pretreatment) are sufficient for a $90 \%$ power to statistically demonstrate an increase of testicular volume following the addition of corifollitropin alfa to hCG treatment. In addition, when either the increase from Day 1 is somewhat smaller (e.g., 0.45 on log-scale) or the variation is somewhat larger (e.g., 0.5 on log-scale), the power is close to $90 \%$.

Efficacy analyses were based on the Full Analysis Set population, including all subjects who received any dose of corifollitropin alfa and who had a baseline and at least one post-baseline measurement of the efficacy variable in question. The mean change from Day 1 (first day of the combined treatment phase) in log-transformed testicular volume was analyzed using a mixed model with a fixed-effect for time point and a random effect for subject. The mixed model accounted for missing data, assuming that data were missing at random. In addition, Last Observation Carried Forward approaches were performed for sensitivity analyses. For each time point (i.e., Weeks 4 to 52), the mean change from Day 1 to that time point and the associated $95 \%$ CI were calculated. The geometric mean increase in testicular volume and its $95 \%$ CI were obtained by exponentiation. For secondary efficacy endpoints, summary statistics included the number of observations, mean, SD, median, minimum, and maximum for continuous variables; frequency distributions were provided for categorical variables. For endocrine parameters, values below the lower limit of quantitation (LLOQ) were set to $0.5 \times$ LLOQ for the calculation of descriptive statistics. For each parameter, descriptive statistics were calculated per assessment, including the 'last measurement' assessment and the minimum and maximum post-baseline value. The calculations were also performed for the absolute and percentage changes from baseline.

Safety analyses were based on all subjects treated, including all subjects who received any dose of corifollitropin alfa. Summary statistics and/or incidence rates were provided for descriptive safety endpoints including AEs, deaths, laboratory parameters, vital signs, and body weight.

\section{Results \\ Disposition of subjects}

The flow of participants through the study is illustrated in Additional file 2: Figure S2. All subjects were white males who ranged in age from 20 to 50 years (mean: 31.5 years). The mean weight of subjects was $84.0 \mathrm{~kg}$ and the mean BMI was $26.4 \mathrm{~kg} / \mathrm{m}^{2}$. In the $14 / 18$ men with a hypothalamic cause of hypogonadism, 11 presented with Kallmann syndrome, 2 were diagnosed with idiopathic $\mathrm{HH}$, and 1 had other causes for $\mathrm{HH}$. In the 4/18 men with a pituitary cause of hypogonadism, 3 subjects were diagnosed with panhypopituitarism ( 1 with coincident pituitary tumor) and 1 had pituitary malformation. In the overall population, 1 subject had received previous treatment with $\mathrm{GnRH}$ and 7 subjects with gonadotropins.

\section{Testicular size}

Over the 52-week combined treatment phase, the mean testicular volume $( \pm \mathrm{SD})$ increased from 8.6 $( \pm 6.09)$ to $17.8( \pm 8.93)$, with a geometric mean fold increase of 2.30 (95\% CI: 2.03, 2.62). Starting from Week 4, the mean testicular volume increased and stabilized after Week 16 of the 52-week combined treatment phase (Fig. 1). The results for the left and right testes were consistent with that of total testicular volume (data not shown). During this period, 7/18 (39\%) subjects had the twice-weekly dose of hCG uptitrated from 1500 IU to $3000 \mathrm{IU}$.

\section{Induction of spermatogenesis}

Fourteen (77.8\%) subjects achieved a sperm count of $\geq 1 \times 10^{6} / \mathrm{mL}$ at one of their assessments from the Day 1 


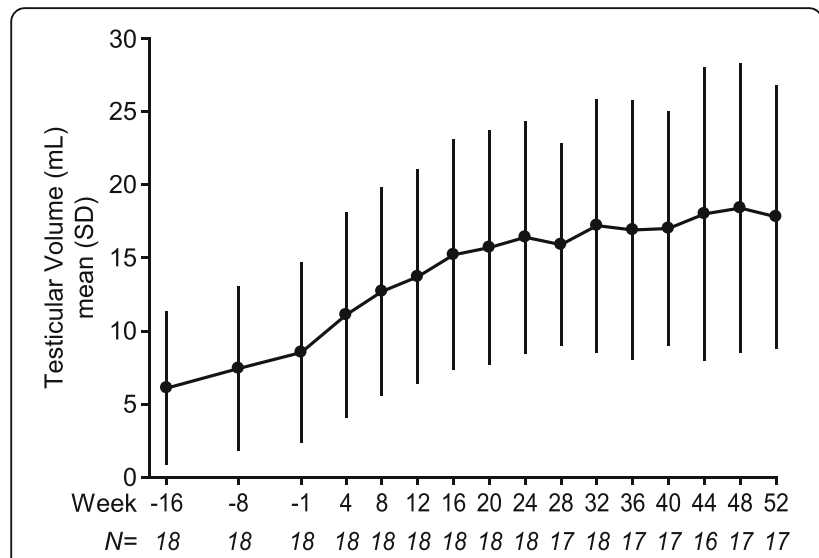

Fig. 1 Mean $( \pm S D)$ testicular volume at each study visit from pretreatment baseline to Week 52 of the combined treatment phase

exam at the start of corifollitropin alfa therapy in the combined treatment phase up to Week 52 (i.e., the end of the combined treatment phase). The mean sperm concentration increased from 0 at Day 1 to $5.2 \times 10^{6} / \mathrm{mL}$ at Week 52 (Fig. 2a). The total sperm count over the course of the combined treatment phase is illustrated in Fig. 2b. By the end of the combined treatment phase, an

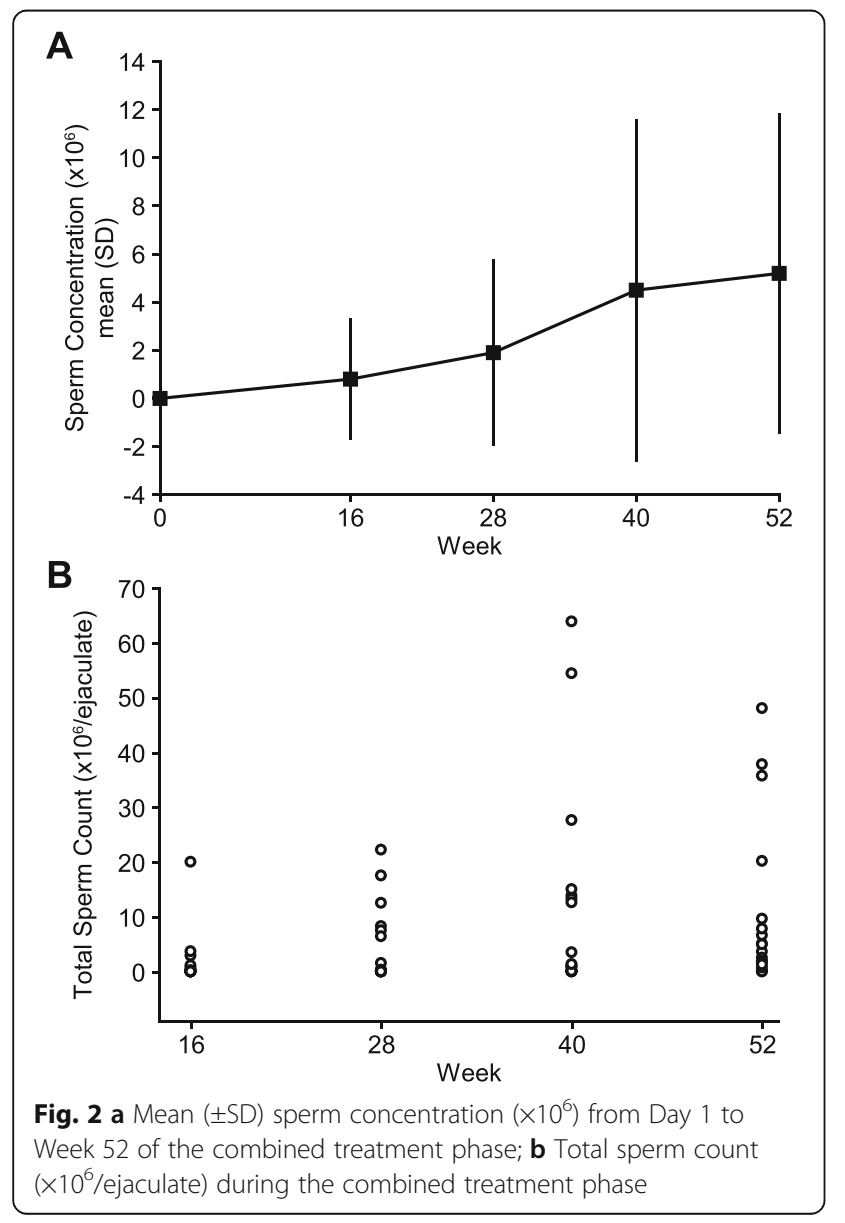

overall mean fraction $( \pm \mathrm{SD})$ of $26.4 \%( \pm 15.6 \%)$ of progressively motile sperm cells were observed, and the overall mean percentage of morphologically normal sperm cells was $11.4 \%$ ( $\pm 18.5 \%)$ (Fig. 3 ).

\section{Hormonal responses}

Figure 4 illustrates the serum hormone levels from baseline to Week 52 of the combined treatment phase. During the 16-week hCG-only pretreatment phase, mean serum $\mathrm{T}$ levels increased from $65.2 \mathrm{ng} / \mathrm{dL}$ to $437.6 \mathrm{ng} / \mathrm{dL}$; men who continued into the combined treatment phase had an additional mean increase of $297.8 \mathrm{ng} / \mathrm{dL}$ (from $409.7 \mathrm{ng} / \mathrm{dL}$ at Day 1 to $669.8 \mathrm{ng} / \mathrm{dL}$ at Week 52). E2 levels rose from 6.8 to $25.6 \mathrm{pg} / \mathrm{mL}$ in the pretreatment phase, with an additional mean increase of $13.4 \mathrm{pg} / \mathrm{mL}$ by Week 52 in the combined treatment phase. FSH levels did not change during the pretreatment phase of the trial, and increased from a median of $0.5 \mathrm{mIU} / \mathrm{mL}$ on Day 1 to $6.6 \mathrm{mIU} / \mathrm{mL}$ by Week 52 in the combined treatment phase. Most of this increase occurred during the first 8 weeks, after which time FSH levels remained stable to the end of the study and then decreased to near Day 1 levels $(0.5 \mathrm{mIU} / \mathrm{mL})$ by the final follow-up visit when treatment had been discontinued ( $\geq 21$ days after the last dose of corifollitropin alfa and

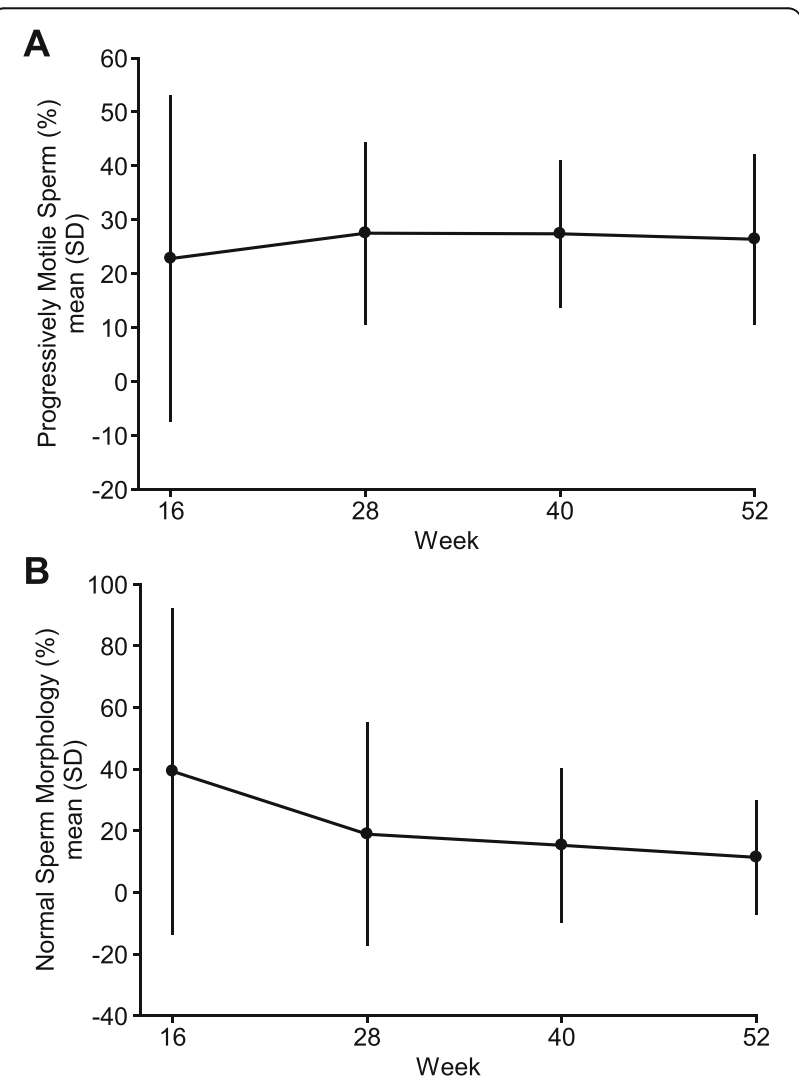

Fig. 3 a Mean $( \pm S D)$ percentage of progressively motile sperm during the combined treatment phase; $\mathbf{b}$ Mean $( \pm \mathrm{SD})$ percentage of normal morphology sperm during the combined treatment phase 

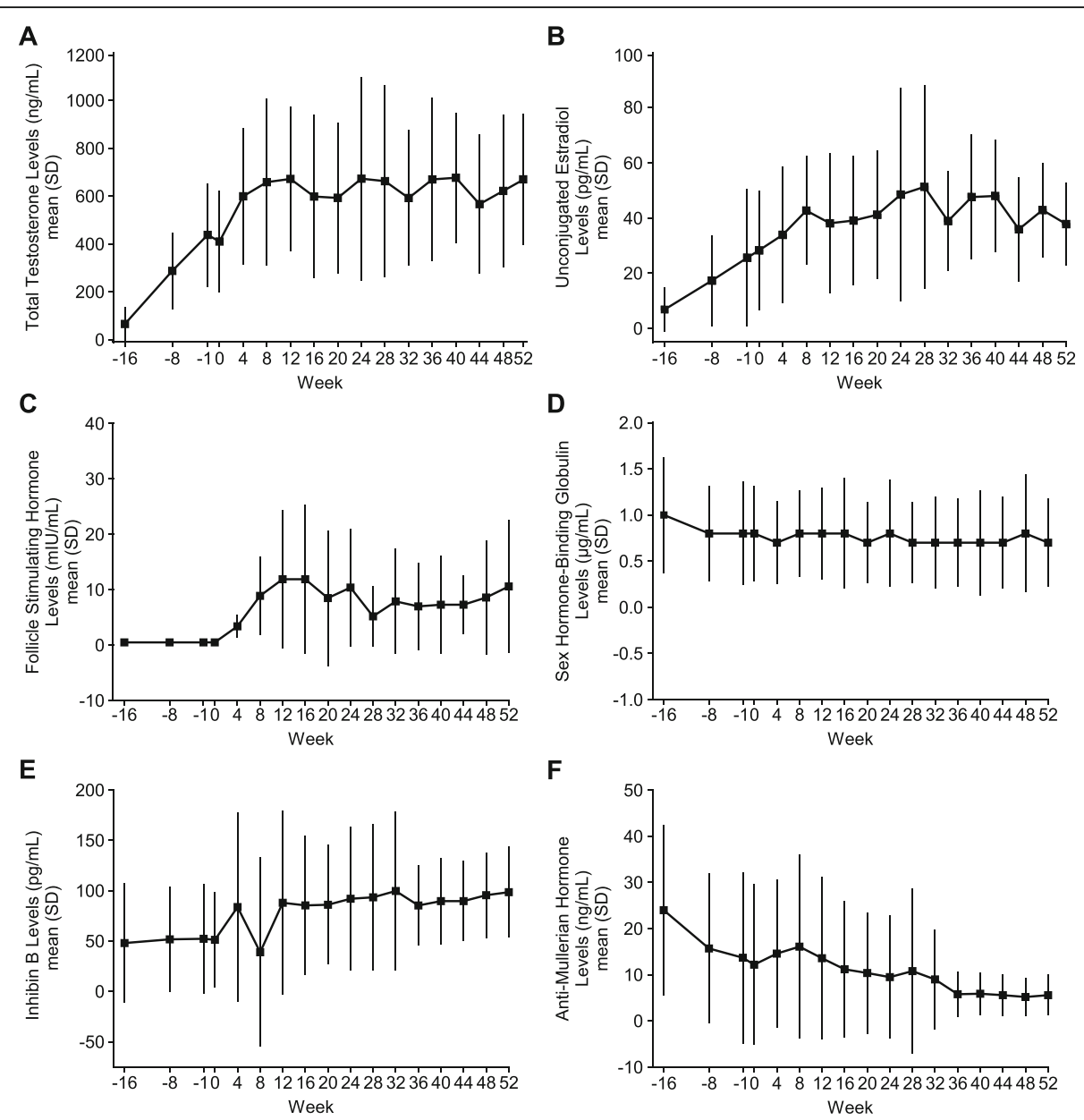

Fig. 4 a-f Mean $( \pm S D)$ hormone levels from pretreatment baseline to Week 52 of the combined treatment phase

$\geq 7$ days after the last dose of hCG). Mean serum SHBG levels remained fairly constant over the treatment period. Inhibin-B levels remained relatively stable during the hCGonly pretreatment phase, but showed a mean increase during the combined treatment phase, from $51.3 \mathrm{pg} / \mathrm{mL}$ $( \pm 46.92)$ on Day 1 to $98.6 \mathrm{pg} / \mathrm{mL}( \pm 44.28)$ at Week $52(1.9$ fold increase). Mean AMH levels declined slightly during the pretreatment phase of the trial, from 24.0 to $13.7 \mathrm{ng} / \mathrm{mL}$, and a greater drop ( $>2$-fold) occurred during the combined treatment phase. The levels of LH did not change during either the pretreatment phase or the combined treatment phase (data not shown).

\section{Safety}

No subject developed confirmed anti-corifollitropin alfa antibodies during this trial. A total of 11 (61.1\%) subjects had one or more AEs during the combined treatment phase (Additional file 3: Table S1). The most frequently reported AEs were nasopharyngitis (22.2\%), estradiol increased $(16.7 \%)$, and headache $(16.7 \%)$; the remaining AEs were reported in two or fewer subjects. Five (27.8\%) subjects had AEs that were considered by the investigators to be drug-related. The most frequently reported drug-related AEs were estradiol increased $(n=3)$, blood testosterone increased $(n=2)$, and blood testosterone decreased $(n=2)$; there were no other AEs considered to be drug-related reported in more than one subject during the combined treatment phase. There were no SAEs and no deaths reported during the combined treatment phase. One subject was discontinued from the trial during the combined treatment phase because of an $\mathrm{AE}$ (blood testosterone increased to $1528 \mathrm{ng} / \mathrm{dL}$ at Week 32), which was considered by the investigator to be drug-related. No clinically relevant changes in laboratory values or vital signs were observed.

\section{Discussion}

Combined treatment with corifollitropin alfa and hCG for 52 weeks resulted in a greater than two-fold increase in testicular volume in men with $\mathrm{HH}$ who remained azoospermic after 16 weeks of treatment with hCG treatment alone, meeting the primary hypothesis of the 
study. Treatment with corifollitropin alfa resulted in an increase in the sperm count of $\geq 1 \times 10^{6} / \mathrm{mL}$ at or before week 52 of the combined treatment phase in 14 of 18 (77.8\%) men, meeting the key secondary hypothesis of the study. No subject developed antibodies to corifollitropin alfa. Treatment was generally well tolerated and no new safety signals were identified during the conduct of this study.

$\mathrm{HH}$ is a rare disease affecting both men and women. In men, the lack of endogenous FSH and LH results in insufficient testicular hormone production and lack of spermatogenesis. The current treatment of pre- or postpubertal $\mathrm{HH}$ to achieve virilization and fertility requires three-times-weekly injections of gonadotropins $(2 \times$ hCG and $3 \times$ recFSH). Whereas combined treatment with hCG and recFSH has been successful for the treatment of $\mathrm{HH}[6,13-16]$, the use of corifollitropin alfa in place of recFSH would reduce the number of required injections and may improve patient satisfaction and compliance. This is important, as treatment for infertility is often prolonged and may require up to two years of continuous treatment [17].

Corifollitropin alfa resulted in an increase in Total T, E2, and inhibin-B levels, and a corresponding decrease in $\mathrm{AMH}$ levels. These changes are consistent with what is expected prior to the onset of spermatogenesis. The increase in $\mathrm{T}$ was slightly less than that observed in the recFSH trial, but this did not appear to negatively impact the primary or key secondary outcomes of the trial. The FSH levels, endogenous only at baseline, endogenous plus corifollitropin alfa levels at Week 52 , increased during the first 8 weeks of the combined treatment phase, remained relatively stable until Week 52, and returned to near Day 1 levels following treatment discontinuation. This pattern is consistent with the expected outcomes of this trial. All other laboratory values, except for AMH which was not measured in the recFSH trial, were similar to those observed previously with recFSH [6].

Following the changes in serum hormone levels, sperm counts increased in 14 of 18 subjects during the last half of the trial. This finding is consistent with the time required to complete spermatogenesis. Assuming an immediate response to corifollitropin alfa treatment, sperm would not be expected to appear in seminal fluid before approximately 75 days after initiation of treatment in an azoospermic male. The increase in the percent of sperm with abnormal morphology over the course of the trial may be related to the increased number of sperm available for assessment from men who were azoospermic at baseline, especially considering that the range of morphologically abnormal sperm is $52-97 \%$ in healthy, fertile men [12]. A similar increase (from 74.5-93.2\%) in the percentage of sperm with abnormal morphology was observed in an earlier trial in men with $\mathrm{HH}$, in which spermatogenesis was induced with hCG and recFSH [6]; thus, the present finding is not unexpected.

The increases in testicular volume and sperm counts observed in the present study suggest that corifollitropin alfa can effectively replace recFSH in the treatment regimen of adult men with $\mathrm{HH}$ desiring fertility. The target sperm count of at least $1 \times 10^{6} / \mathrm{mL}$ was selected based on comparable studies showing that such concentrations can realistically be attained within the time frame of a clinical trial involving $\mathrm{HH}$ patients. For instance, in a previous study of recFSH and hCG for the treatment of $\mathrm{HH}, 47 \%$ of men achieved sperm counts $\geq 1 \times 10^{6} / \mathrm{mL}$ [6]. In the current study, nearly $78 \%$ of men treated with corifollitropin alfa and hCG reached this threshold. The sustained elevation in serum FSH levels resulting from corifollitropin alfa injections most likely contribute to the high success rate of this regimen. Although sperm concentrations and counts remained in the low range, it should be noted that the sperm produced by these men would be expected to have a high capacity to fertilize eggs, either spontaneously or by intracytoplasmic sperm injection (ICSI), as pregnancy rates of $65-90 \%$ in previous studies have been shown $[4,14,18]$. While the chances for paternity in corifollitropin alfa-treated $\mathrm{HH}$ patients should be high based on data from these previous studies, it was not one of the endpoints examined in the present study.

Adverse events were reported for 11 (61.1\%) subjects who received corifollitropin alfa. The investigators considered the AEs to be drug-related in 5 of these 11 subjects. The most commonly reported AEs were related to changes in serum hormone levels (increasing or decreasing $\mathrm{T}$, or an increase in E2), and none was reported as an SAE. One subject was discontinued from treatment because of an increase in the Total $\mathrm{T}$ level to $1528 \mathrm{ng} / \mathrm{dL}$. This level was reversible and declined after discontinuation of treatment. The event was judged by the investigator as likely to be drug-related. There were no deaths reported in the trial. These findings support the overall safety of corifollitropin alfa.

This study had several limitations. This was a single-arm study that did not employ a control group. In addition, the number of men included in this study was small. Finally, sperm morphologies were performed locally, a test that might introduce variability across laboratories; however, all sites were trained with the standardized methodology prior to the start of the study to mitigate the potential for such variability.

\section{Conclusions}

In conclusion, in men with $\mathrm{HH}$ pretreated with hCG alone for 16 weeks, addition of corifollitropin alfa to ongoing hCG treatment resulted in more than a doubling in testicular volume and an increase in sperm count to at 
least $1 \times 10^{6} / \mathrm{mL}$ in 14 of 18 men, with appropriate increases in total T, E2, and inhibin B, and a decrease in $\mathrm{AMH}$ levels. In addition, there were no new safety signals identified, with no development of anti-corifollitropin alfa antibodies. Taken together, these results suggest that longacting corifollitropin alfa can effectively and safely replace recFSH in the treatment regimen of adult men with $\mathrm{HH}$ desiring fertility. Additional studies exploring the role of corifollitropin alfa in the treatment of male $\mathrm{HH}$ are warranted.

\section{Additional files}

Additional file 1: Figure S1. Study design. (EPS $1160 \mathrm{~kb}$ )

Additional file 2: Figure S2. Subject disposition. (EPS $1138 \mathrm{~kb}$ )

Additional file 3: Table S1. Summary of Adverse Events in the Combined Treatment Phase. (DOCX 29 kb)

\begin{abstract}
Abbreviations
ABL: Analytical Biochemical Laboratory; AEs: Adverse events; ALT: Alanine aminotransferase; AMH: anti-Müllerian hormone; ART: Assisted reproductive technology; AST: Aspartate aminotransferase; Cl: Confidence interval; E2: Estradiol; ElA: Enzyme immunoassay; FSH: Follicle-stimulating hormone; GnRH: Gonadotropin-releasing hormone; hCG: Human chorionic gonadotropin; HH: Hypogonadotropic hypogonadism; hMG: Human menopausal gonadotropin; ICSI: Intracytoplasmic sperm injection; LH: Luteinizing hormone; LLOQ: Lower limit of quantitation; PD: Pharmacodynamic; recFSH: Recombinant follicle-stimulating hormone; SAEs: Serious adverse events; SC: Subcutaneously; SD: Standard deviation; SHBG: Sex hormone-binding globulin; T: Testosterone; ULN: Upper limit of normal
\end{abstract}

\section{Acknowledgments}

The authors thank Jennifer Rotonda PhD and Michele McColgan BA of Merck \& Co., Inc., Kenilworth, NJ, USA for editorial assistance.

\section{Funding}

Funding for this study was provided by Merck \& Co., Inc., Kenilworth, NJ, USA.

\section{Availability of data and materials}

To protect the privacy and confidentiality of research participants, there are restrictions on the availability of data from this study (See Merck \& Co., Inc. data sharing policy at: http://engagezone.merck.com/ds_documentation.php). Requests for access to the study data can be submitted through the EngageZone Web site or via email to dataaccess@merck.com.

\section{Authors' contributions}

EN, PMGB, BJS, RRS, YG, AT, CMS, and HMB are responsible for the work described in this paper. All authors were involved in at least one of the following: conception, design, acquisition, analysis, statistical analysis, and interpretation of data in addition to drafting the manuscript and/or revising/reviewing the manuscript for important intellectual content. All authors read and approved the final manuscript.

\section{Competing interests}

EN has received consulting fees and travel support from MSD. PMGB has nothing to declare. BJS was previously employed by Merck Sharp \& Dohme Corp., a subsidiary of Merck \& Co., Inc., has equity interests in Merck \& Co., Inc., and has also served as a consultant to Merck \& Co., Inc. RRS, YG, AT, and CMS are employed by Merck Sharp \& Dohme Corp., a subsidiary of Merck \& Co., Inc., and have equity interests in Merck \& Co., Inc. HMB has received consulting fees and travel support from MSD, and his institution has received grant support.

\section{Consent for publication}

All authors provided final approval of the version to be published and agree to be accountable for all aspects of the work in ensuring that questions related to the accuracy or integrity of any part of the work are appropriately investigated and resolved.

\section{Ethics approval and consent to participate}

The study protocol was approved by the institutional review boards at every study center and informed consent was obtained before the initiation of any study procedures.

\section{Study investigators}

The Primary Study Investigators for Protocol MK-8962-031-01 (by country) were: David Handelsman (Australia); Robert McLachlan (Australia); Hermann M Behre (Germany); Sabine Kliesch (Germany); Alessandro Pizzocaro (Italy); Andrea Lenzi (Italy); Mario Maggi (Italy); Waldemar Kuczynski (Poland); Katarzyna Koziol (Poland); Miguel Ruiz Jorro (Spain); Lluis Bassas (Spain); Pierre Bouloux (UK); Richard Quinton (UK).

\section{Author details}

${ }^{1}$ University Hospital of Muenster, Center of Reproductive Medicine and Andrology, Domagkstraße 11, D-48149 Muenster, Germany. ${ }^{2}$ The Royal Free Hospital, Pond St, London NW3 2QG, UK. ${ }^{3}$ Merck \& Co., Inc., 2000 Galloping Hill Road, Kenilworth, NJ 07033, USA. ${ }^{4}$ University Hospital Halle (Saale), Martin Luther University Halle-Wittenberg, Ernst-Grube-Str. 40, D-06120 Halle, Germany.

Received: 18 January 2017 Accepted: 21 February 2017

Published online: 07 March 2017

\section{References}

1. Fraietta R, Zylberstejn DS, Esteves SC. Hypogonadotropic hypogonadism revisited. Clinics (Sao Paulo). 2013;68 Suppl 1:81-8.

2. Rohayem J, Zitzmann M, Nieschlag E. Congenital hypogondotropic hypogonadism and Kallmann's syndrome. Reference Module in Biomedical Sciences. Elsevier 12-Dec-15; doi:10.1016/B978-0-12-801238-3.98874-3.

3. Lytton B, Kase N. Effects of human menopausal gonadotrophin on a eunuchoidal male. N Engl J Med. 1966;274:1061-4.

4. Buechter D, Behre HM, Kliesch S, Nieschlag E. Pulsatile GnRH or human chorionic gonadotropin/human menopausal gonadotropin as effective treatment for men with hypogonadotropic hypogonadism: a review of 42 cases. Eur J Endocrinol. 1998;139:298-303.

5. Kliesch S, Behre HM, Nieschlag E. Recombinant human follicle-stimulating hormone and human chorionic gonadotropin for induction of spermatogenesis in a hypogonadotropic male. Fertil Steril. 1995;63:1326-8

6. Bouloux PMG, Nieschlag E, Burger HG, Skakkebaek NE, Wu FCW, Handelsman DJ. Induction of spermatogenesis by recombinant folliclestimulating hormone (Puregon) in hypogonadotropic azoospermic men who failed to respond to human chorionic gonadotropin alone. J Androl. 2003:24:604-11.

7. Corifollitropin alfa Ensure Study Group. Corifollitropin alfa for ovarian stimulation in IVF: a randomized trial in lower-body-weight women. Reprod Biomed Online. 2010;21:66-76

8. Devroey P, Boostanfar R, Koper NP, Mannaerts BM, ljzerman-Boon PC, Fauser BC. A double-blind, non-inferiority RCT comparing corifollitropin alfa and recombinant FSH during the first seven days of ovarian stimulation using a GnRH antagonist protocol. Hum Reprod. 2009;24:3063-72. Corrigendum: Hum Reprod. 2014;29:1116-20.

9. Boostanfar R, Shapiro B, Levy M, Rosenwaks Z, Witjes H, Stegmann BJ, et al. Large, comparative, randomized double-blind trial confirming noninferiority of pregnancy rates for corifollitropin alfa compared with recombinant follicle-stimulating hormone in a gonadotropin-releasing hormone antagonist controlled ovarian stimulation protocol in older patients undergoing in vitro fertilization. Fertil Steril. 2015;104:94-103.

10. Fauser BCJM, Mannaerts BMJL, Devroey P, Leader A, Boime I, Baird DT. Advances in recombinant DNA technology: corifollitropin alfa, a hybrid molecule with sustained follicle-stimulating activity and reduced injection frequency. Hum Reprod Update. 2009;15:309-21.

11. Bouloux PMG, Handelsman DJ, Jockenhövel F, Nieschlag E, Rabinovici J, Frasa WLH. First human exposure to FSH-CTP in hypogonadotropic hypogonadal males. Hum Reprod. 2001;16:1592-7.

12. WHO Laboratory Manual for the Examination and Processing of Human Sperm, 5th edition. Geneva: World Health Organisation; 2010. 
13. Burgués S, Calderón MD, the Spanish Collaborative Group on Male Hypogonadotropic Hypogonadism. Subcutaneous self-administration of highly purified follicle stimulating hormone and human chorionic gonadotrophin for the treatment of male hypogonadotrophic hypogonadism. Hum Reprod. 1997;12:980-6.

14. European Metrodin HP Study Group. Efficacy and safety of highly purified urinary follicle-stimulating hormone with human chorionic gonadotropin for treating men with isolated hypogonadotropic hypogonadism. Fertil Steril. 1998;70:256-62.

15. Liu PY, Turner L, Rushford D, McDonald J, Gordon Baker HW, Conway AJ, et al. Efficacy and safety of recombinant human follicle stimulating hormone (Gonal-F) with urinary human chorionic gonadotrophin for induction of spermatogenesis and fertility in gonadotrophin-deficient men. Hum Reprod. 1999;14:1540-5.

16. Rohayem J, Hauffa BP, Zacharin M, Kliesch S, Zitzmann M, German Adolescent Hypogonadotropic Hypogonadism Study Group. Testicular growth and spermatogenesis: new goals for pubertal hormone replacement in boys with hypogonadotrophic hypogonadism? A multicentre prospective study of hCG/rFSH treatment outcomes during adolescence. Clin Endocrinol (Oxf). 2017;86:75-87.

17. Rocchietti March M, Isidori A. New frontiers in the treatment of male sterility. Contraception. 2002;65:279-81.

18. Rohayem J, Sinthofen N, Nieschlag E, Kliesch S, Zitzmann. Causes of hypogonadotropic hypogonadism predict response to gonadotropin substitution in adults. Andrology. 2016;4:87-94.

\section{Submit your next manuscript to BioMed Central and we will help you at every step:}

- We accept pre-submission inquiries

- Our selector tool helps you to find the most relevant journal

- We provide round the clock customer support

- Convenient online submission

- Thorough peer review

- Inclusion in PubMed and all major indexing services

- Maximum visibility for your research

Submit your manuscript at www.biomedcentral.com/submit

) Biomed Central 